\title{
HEDGING STRATEGIES IN RESEARCH ARTICLES: A COMPARATIVE ANALYSIS OF INDONESIAN MALE AND FEMALE ENGLISH STUDENTS
}

\author{
Ade Windiana Argina ${ }^{1}$, Nur Ijabah ${ }^{2}$ \\ ${ }^{1}$ Politeknik LP3I Jakarta \\ ${ }^{2}$ Universitas Trunojoyo Madura \\ Email: adewindianaa@student.upi.edu
}

\begin{abstract}
Hedge is claimed as linguistic of full commitment or precision commonly used in academic writing to give the appropriate uncertainty, proper strength of claimed data, politeness, and precise presentation in providing and presenting literary works i.e. research articles. This study aimed at revealing the tendency and preferences of Indonesian English Students in applying hedging strategies, and frequencies of hedges use, and if there were any differences between males and females in using hedges in research articles. Data corpus was composed of 40 articles written in English by male and female postgraduate students majoring English Education. The output was categorized which was referred to a combined framework proposed by Holmes (1988), Hyland (1998), Hyland and Milton (1997), Vartala (2001), and Crompton (1997) covering 9 types of hedges. The statistical result showed that although Indonesian Male English Students (IEMSs) employed Hedges in their research articles more frequently than Indonesian Female English Students (IEFSs) did, MANOVA proved that there was no significant effect of gender on the use of hedges in research articles written by Indonesian English Students. Nevertheless, ANOVA result confirmed significant main effects of gender on the use of some types of hedge (Adverb of Frequency, Quantifiers, Epistemic Lexical Verbs and Adjective\& Adverb Informal).
\end{abstract}

Keywords: gender, hedges, Indonesian-English students

(c) (i) This work is licensed under Creative Commons Attribution License 4.0 CC-BY International license 


\section{INTRODUCTION}

\subsection{Introduction}

The different use of language by men and women has become a great interest for language practitioners, specifically discourse analysis. Even though men and women live in the same environment, they construct different relation and intimacy with society as if each of them belongs to a different culture (Nemati \& Bayer, 2007). Lastly, based on the theory of social constructionist, language that shapes and is shaped by gender is claimed as a social phenomenon.

Another assumption proposed by Lakoff (1975) is that women have a lack of authority because, in order to become feminine, women must learn to adopt an unassertive style of communication. Based on Lakoff, women's language refers to a group of linguistic devices that serve functions including hesitations, intensive adverbs, empty adjectives, tag questions, compound requests, and also hedges.

Swales (1990), hedging has been unarguably a critical issue for all levels of academic writing. Hyland (1994) tried to actualize his attention on hedges by indicating the importance and essential of hedges in even textbooks on top of academic writing. As Hyland confirmed (1998) this importance brings hedged as labeled as the most frequent features of writer perspective. It has been strengthened by Jordan (1997) claiming that hedge is a tool in which its existence is in academic writings as requisite. It is reasonable since the scientific study has been inevitably bound up with “exactness'. It is a media of scientific writers to explore and deliver their insight explicitly and precisely. Yet, there might some reasons of some writers not to be exact and explicit; for instance, considering the consequences of inadequacy in their scientific claims or to express their uncertainty about scientific issues or reflect the state of knowledge. Brown and Levinson (1988) argued that presenting a scientific claim is a face-threatening act, so even if the scientific writer is utterly certain about his claims to be presented explicitly and precisely with no anxiety for the later critics, some degree of uncertainty and fussiness is often applied, to leave a little space for readers. It defines that scientific writing does not only deal with the content, matter, or findings of a study or research writing, but the way it is presented is a significant process to have positive acceptances from the readers of the scientific community. Hence, applying hedges strategy is a device composed of several types with different objectives that help researchers appropriately express scientific claims. 
According to Hinkel (2003), hedging is not obvious consideration for many non-native writers compared to native writers. It is strengthened by Hyland (2002) alleging that non-native English writers have a restricted lexical repertoire of hedging devices in their L2 writings.

English postgraduate students in Indonesia have obviously dealt with a tremendous number of scientific research. Yet, research does not only engage with the content, methods, and results of a study but also with the way it is presented to grab readers' positive acceptance. It also strongly counts on how all of the elements of the study are presented and how the researchers position themselves on the study. One way to arrive at those points is by employing hedges. From then on, it sounded to conduct a study focusing on hedges used in academic writing, research articles, by non-native English learners, specifically for EFL students like Indonesian students of English by considering gender differences.

\subsection{Research questions}

This study aims at reaching the following objectives: (1) to examine the overall frequency of hedges used in research articles written by Indonesian male and female English students; (2) to compare the frequency and the distribution of hedges used in research articles written by Indonesian male and female English students from Introduction, Discussion, and Conclusion sections; 3) to identify whether or not gender significantly affect the different number of the hedges used in research articles written by Indonesian male and female English students.

\subsection{Significance of the study}

Theoretically, this study is expected to enrich the sources and references related to language use in academic writing especially the use of hedging strategies in research articles. Practically, the findings in this study might also be used as a reflection for researchers to empower their writing especially in presenting the results of their studies. In addition, the findings of this study can also be professionally used as a reference by the English practitioners being attracted to academic writing and hedges in developing their learning materials and in promoting the importance of the use of hedging strategies in writing.

\section{METHOD}

\subsection{Research Design}

Considering the research questions addressed, this study used a quantitative descriptive design in order to statistically present the tendency and preferences of Indonesian English Students in

applying hedging strategies, and frequencies of hedges use, and differences between males and females in using hedges in research articles (if any). After the data gathered (see 2.2) and 
analyzed using ANOVA and MANOVA (see 2.4), the findings are statically presented and described to help the readers understands how the findings answer the research questions.

Quantitatively, this study employed MANOVA and ANOVA

\subsection{Samples/Participants}

A total of 40 research articles written by Indonesian English students (20 males and 20 females) constituted the data for this study. The research articles were selected randomly from various journals of English Language Teaching (ELT). This study only focused on English postgraduate students as stated by Hyland (2005) hedges and other features of language used in delivering opinion is said to be different across disciplines. The analyzed research articles were research articles published in the last 5 years in order to view the variation of the use of hedges. In selecting the research articles, it also considered the equal length of the research articles in purposing to keep the balance of the percentage of the hedges employed.

\subsection{Instruments}

The taxonomy used in this study was the hedging devices or types based on their own function. The types of hedges used as the indicators were 9 types of hedges proposed by several scholars (Holmes, 1988; Hyland, 1998; Hyland and Milton,1997; Vartala, 2001; and Crompton,1997):

1. Adverbs of frequency: frequently, usually, often, occasionally, rarely...

2. Quantifiers: some, a few, a bit, a good deal, many...

3. Epistemic modality verbs: can, may, might, could, be able to, must, should, need to, to be to, will, would..

4. Epistemic lexical verbs: to seem, to appear, to believe, to assume, to suggest, to estimate, to tend to, to think, to indicate, to argue, to purpose, to speculate

5. Adjectives and Adverbs:

a) Formal: slightly, relatively, somehow, presumably, actually, merely, eventually...

b) Informal: almost, (a) little, (a) few, enough, only, quite, pretty, basically, at least....

6. Nouns: claim, suggestion, assumption, estimate, possibility.....

7. Conversational \&Informal: kind of, sort of, anyway, in a way, more or less, maybe, like..

8. Introductory phrases: it is our view that, we feel that, we think that...

9. Vague references: as we know, as we all know, as it is known, as people say, as the readers knows. 


\subsection{Data analysis}

In demonstrating the hedges, the analysis was conducted by scanning the whole devices in each research article by using feature "finding" provided in Microsoft Word. Yet, it was needed to do another scanning since this program categorizes the vocabularies only in terms of their frequency, not in terms of semantics and pragmatics. Furthermore, in analyzing the devices, the whole context where a device (s) was (were) detected was looked at deeply not just by counting the isolated words or devices of hedges. It is because as stated by Holmes (1990), researchers should avoid pointing out or analyzing the hedges type only by looking at the frequency of the occurrences of certain words since each word can reflect a different function. As the purpose of this study was to draw the distribution pattern of hedges used by English male and female students across sections in their research articles, each section was analyzed including Introduction, Discussion, and Conclusion. To answer the research question related to comparing whether or not there was a significant difference between the number of hedges used by English male and female students in their research articles, a descriptive statistic was employed. MANOVA was applied to investigate whether or not the gender of the writer affects the use of hedges types. Hence, the series of univariate ANOVA analyses were also conducted to identify the significant main effect for each dependent variable (each type of hedges) to the independent variable (gender).

\section{FINDINGS AND DISCUSSION}

\subsection{Findings}

\subsubsection{Hedging across Research Article Section}

\section{Figure 1. Hedging distribution across section}

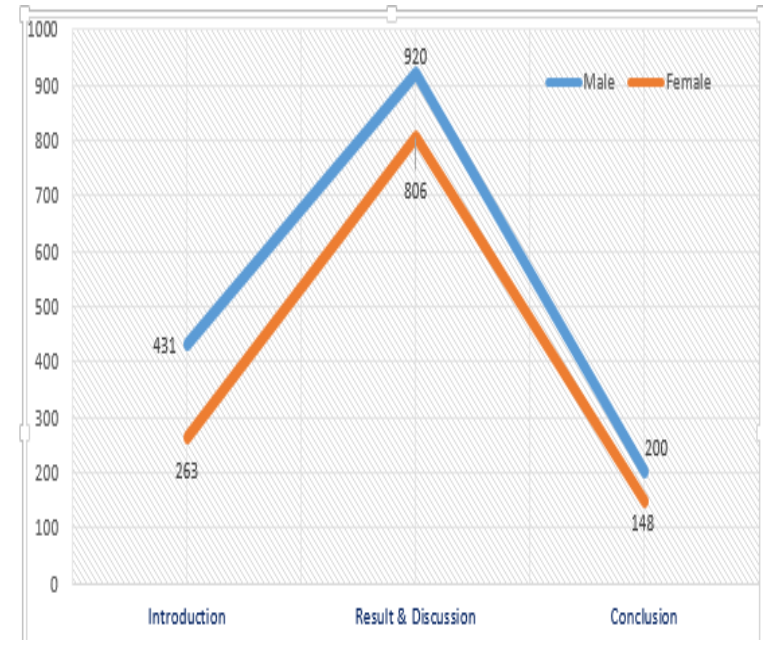


As shown by the figure above, the inclinations of the use of hedges across section by male and female students are similar. The most frequently appeared hedges both in male and female students' research articles are in the section of Result and Discussion, then followed by the section of introduction and the least number of the use of hedges appeared in the section of Conclusion. Deeply, IMES employed hedges over 200 in Introduction while over 400 were employed by IFES. Whereas, it is clearly shown that the section of Result and Discussion in which both IFES and IMES used hedges the most; over 920 for IMES and 800 for IFES. It indicates that there is a comparative disposition of IMES in using hedging devices in the section of Result and Discussion. For introduction, the figure captures the same in which IMES used more hedges than IFES did. It also clearly shows the tendency of IMES to use hedges more than IFES in all sections. Meanwhile, to be compared, the difference between the section of Result \& Discussion and Conclusion is slighter than in Introduction section is. It is proved by IMES used 168 more hedge devices than IFES did.

\subsubsection{Hedge Type across Section}

Data shows that IMESs more frequently use type of hedges including Adverb of Frequency, Quantifiers, Epistemic Modality Verbs, Adjective and Adverbs formal, Nouns, Conversational\& Informal and Vague References, while IFESs more frequently use Adjectives and Adverbs Informal and Introductory Phrases. Epistemic Modality Verbs gains the highest number of the employment with 2418. Among all types, the least use is Introductory Phrases with 8. Both IMESs and IFESs presents the similar frequency of the use of hedging types meaning that both of them used Epistemic Modality Verbs the most while Introductory Phrases the least. Moreover, both of the groups showed slighter difference from one to another in hedge types such as Adjectives and Adverbs Formal, Conversational \& Informal, Introductory Phrases, and Vague References.

In Introduction, both IMESs and IFESs employ Epistemic Modality Verbs as their premier hedging devices with 175 for IMESs and 128 for IFESs. According to the section of Result and Discussion, both IMESs and IFESs employ Epistemic Modality Verbs as their premier hedging devices with 373 for IMESs and 354 for IFESs. And for Conclusion section, Epistemic Modality Verbs is still prominently used by both IMESs and IFESs. 


\subsubsection{The effect of Gender on Use of Hedges}

By employing MANOVA, (with Wilks $\lambda=.612, \mathrm{~F}=33.825, \mathrm{P}=.097$, and $\alpha=.05$ ) it can be concluded that the null hypothesis is accepted meaning that there is no significant multivariate main effect for the gender of the writer on the use of hedges. Yet, it cannot guarantee that gender has no main effect on the use of each type of hedges.

To examine the existence of significant effect of gender on the use of each type of Hedges, this study utilized a series of univariate ANOVA analyses. The results of analysis for each type of hedge are presented as follow with $\alpha(0.05)$ :

Table 1. The significant effect of gender on the use of each type of Hedges

\begin{tabular}{|l|l|c|c|c|}
\hline NO & \multicolumn{1}{|c|}{ Types of Hedges } & F & P & $\alpha(0.05)$ \\
\hline a & Adverb of frequency & 4.335 & 0.004 & $\mathrm{p}<\alpha$ \\
\hline $\mathrm{b}$ & Quantifiers & 4.203 & 0.047 & $\mathrm{p}<\alpha$ \\
\hline $\mathrm{c}$ & Epistemic Modality Verbs & 1.285 & 0.246 & $\mathrm{p}>\alpha$ \\
\hline $\mathrm{d}$ & Epistemic lexical Verbs & 4.766 & 0.035 & $\mathrm{p}<\alpha$ \\
\hline $\mathrm{e}$ & Adjectives and Adverbs Formal & 9.414 & 0.004 & $\mathrm{p}<\alpha$ \\
\hline $\mathrm{f}$ & Adjectives and Adverbs Informal & 0.069 & 0.794 & $\mathrm{p}>\alpha$ \\
\hline $\mathrm{g}$ & Nouns & 2.796 & 0.103 & $\mathrm{p}>\alpha$ \\
\hline $\mathrm{h}$ & Conversational \& Informal & 0.725 & 0.725 & $\mathrm{p}>\alpha$ \\
\hline $\mathrm{i}$ & Introductory Phrases & 0,691 & 0.691 & $\mathrm{p}>\alpha$ \\
\hline $\mathrm{j}$ & Vague References & 0.000 & 0.000 & $\mathrm{P}>\alpha$ \\
\hline
\end{tabular}

a. There is a statistically significant difference between IMESs and IFESs in using Adverb of Frequency as a hedge device

b. There is a statistically significant difference between IMESs and IFESs in using Quantifiers as a hedge device.

c. There is not statistically significant difference between IMESs and IFESs in using Epistemic Modality Verbs as a hedge device. 
d. There is a statistically significant difference between IMESs and IFESs in using Epistemic Lexical Verbs as a hedge device.

$e$. There is a statistically significant difference between IMESs and IFESs in using Adjectives and Adverbs Formal, as a hedge device.

$f$. There is a not statistically significant difference between IMESs and IFESs in using Adjectives and Adverbs Informal as a hedge device

g. There is not statistically significant difference between IMESs and IFESs in using Nouns as a hedge device

h. There is not statistically significant difference between IMESs and IFESs in using Conversational \& Informal as a hedge device

$i$. There is not statistically significant difference between IMESs and IFESs in using Introductory Phrases as a hedge device

j. There is not statistically significant difference between IMESs and IFESs in using Vague References,

\subsection{Discussion}

By considering the tendency of the use of hedge devices, comparatively, IMESs used hedges more frequently than IFESs did in order to ignore absolute statement, to depict their certainty of knowledge accurately; and might be as politeness strategies or it might be other pragmatic personal reasons. This result is in line with Hassani\& Dastjani (2014) and Serholt (2012) which rejects the theory proposed by Lakoff (1970s) claiming that hedges are a part of women's language features. There might be several variable factors affected the use of hedges such as: language, culture, discipline Hyland (2005), and language proficiency Varttala (2001). Yet, even though in total number, IMESs is greater than IFESs, it does not occur in each type of hedge. The data revealed that IFESs lead in some types of hedge, said as Adjectives and Adverbs Informal and Introductory Phrases.

In terms of hedges used in each section, the result of this study is in line with (Hyland \& Milton, 1997; Hyland, 2002), Yagiz and Demir (2014) and Varttala (2001) who found that hedge devices were mostly employed in the section of Result and Discussion while the least number of the hedge usage appear in Conclusion section. It might be caused by the term of Discussion deals with the explanation and judgment of the result of the study that requires a strategy to provide non-rigid judgmental statement, to floor the possibility and avoid the writers' ego in making a 
judge. Moreover, the Section of Result and Discussion contains numerical and not-numerical data that must be interpreted correctly, politely and wisely.

Although this study statistically showed that there is no significant multivariate main effect of gender on the use of hedges in research articles written by Indonesian English Students, the significant main effect for some of hedge types was obviously found such as for Adverb of Frequency, Quantifiers, Epistemic Lexical Verbs and Adjective\& Adverb Informal. Moreover, there is a quite interesting result for the use of vague references. The use of Epistemic Modality Verbs is obviously wide. To be strengthened by Mojica (in Yagiz and Damir, 2014) claiming that Modals, particularly may and might are the favored form of hedging devices. It might be caused by the function of the modality itself as the tool to convey the uncertainty and to avoid selfish judgment.

In this study type of Introductory Phrases is the least used type of hedge by writers, it is in contrast with the study done by Yagiz and Demir (2014) who found that Introductory Phrases is the third widely used among all types. It might be caused by and might be the lack of this study in which rigid determined Introductory Phrases were used as the indicators. When in fact, it was found that a number of Introductory Phrases were found in the research articles written by Indonesian English Students such as: "It is viewed that", "it is felt that", "as we think that", " It is our belief that", "As we believe that" and so on. Hopefully, this weakness can be improved in the nest study.

Thus, this analysis was concluded by statistical results showing that although Indonesian Male English Students employ Hedges in their research articles more frequently than Female English Students did, the significant multivariate main effect measurement using MANOVA proved that there is no significant effect of gender on the use of hedges in research articles written by Indonesian English Students. Yet, there is a significant main effect of gender on the use of each type of hedge that was proven by using univariate ANOVA analysis.

\section{CONCLUSIONS}

\subsection{Conclusion}

This study tried to discover generally the use of hedges by Indonesian Male and Female English students in their research articles across sections namely Introduction, Result and Discussion, and Conclusion. This study also identified the type of hedge used across those sections, analyzed the significant effect of gender on the use of hedges in multivariant variables (all types of hedges), and the significant effect of gender on the use of some types of hedges. 
As stated by Lakoff (1975), hedges are one of the features of women's language that represent their power in society, many studies have been done as a response to her controversial claim. Some scholars were interested in examining the truth value of what Lakoff proposed and some of the results of the study reject the said value as well as this present study. In conclusion of the result of this study, although Indonesian Female English Students were more inclined than males in delivering stronger commitment or perception to the proportional information they supplied, the significant multivariate main effect measurement using MANOVA proved that there is no significant effect of gender on the use of hedges in research articles. Yet, there is a significant main effect of gender on the use of each type of hedge that was proven by using univariate ANOVA analysis.

\subsection{Suggestions}

For further research, it is suggested to conduct a study by adding the types of hedges to be analyzed such as If Conditional and Passive Voice that did not consider in this study. By the lack of this study, it is also recommended for further researchers to use more flexible consideration since sometimes hedges can be found in different word' structures but pragmatically it has the same meaning as hedges.

Since this study only focused on research articles written by Indonesian English postgraduate students, it would be interesting to identify and discover the use of hedges by undergraduate students in their thesis or research articles. Since as briefly mentioned before, the level of English proficiency is claimed as one of several variable factors that affect the use of hedges.

\section{REFERENCES}

Alward, A. (2011). An exploratory study of hedges used in the EFL Yemeni undergraduates' job application letter. Frontiers of Language and Teaching, 280-287.

Alward, A., Mooi, C. C., \& Bidin, J. B. (2012). Hedges and Boosters in the Yemeni EFL Undergraduates' Persuasive Essay: An Empirical Study. The Internet Journal of Language, Culture and Society, 34:1-12.

Behman, B., Naeimi, A., \& Darvishzade, A., (2012). A Comparative Genre Analysis of Hedging Expressions in Research Articles: Is Fuzziness Forever Wicked? English Language and Literature Studies, 2 (2).

Brown, P., \& Levinson, S. (1988). Politeness: Some universals in language usage. Cambridge University Press. 
Chang, M.-H., Luo, Y.-W., \& Hsu, Y.-K. (2012). Subjectivity and Objectivity in Chinese Academic Discourse: How Attribution Hedges Indicate Authorial Stance. Concentric: Studies in Linguistics, 293-329.

Channell, J. (1994). Vague language. Oxford University Press.

Crompton, P. (1997). Hedging in Academic Writing: Some Theoretical Problems. English for Specific Purposes, 16, 4, 271-287.

Dixon, J.A., \& Foster, D.H. (1997). Gender and Hedging: From Sex Differences to Situated Practice. Journal of Psycholinguistics Research, 26 (1), 89-107.

Hassani, M.T., \&Dastjani, F.M. (2014). A Discourse Analysis of gender Differences in The Use of Hedging Devices in Applied Linguistics Research Articles. English Language Teaching, pp.59-73, 1 (1).

Hinkel, E. (2003). Teaching Academic ESL Writing. New York: Routledge.

Holmes, J. (1990). Hedges and Boosters in Women's and Men's Speech. Language \& Communication, 10(3), 185-205.

Holmes, J. (1988). Doubt and Certainty in ESL textbooks. Applied Linguistics, 9 (1), 20-44.

Ha, J. (2008). A Review paper on gender difference in SLA: Sociolinguistics perspective and implications. Modern English Education, 9 (2), 21-24.

Hyland, K. (1994). Hedging in Academic Writing and EAP. English for Specific Purposes, 3: 239-256.

Hyland, K. \& Milton, J. (1997). Qualification and Certainty in L1 and L2 students' writing. Journal of Second Language Writing, 6 (2), 183-205.

Hyland, K. (1998). Hedging in scientific research articles. Amsterdam/Philadelphia: John Benjamins publishing company.

Hyland, K. (2002). Teaching and researching writing. Harlow, Essex: Longman.

Hyland, K. (2005). Stance and Engagement: A Model of Interaction in Academic Discourse. Discourse Studies, 7(2), 173-192.

Jordan, R. (1997). English for Academic Purposes. Cambridge: Cambridge University Press.

Karlsson, S. (2007). Gender-related differences in language use. Available: epubl.ltu.se/14021552/2007/085/LTU-DUPP-07085-SE.pdf.

Lakoff, R. (1972). Hedges: A Study in Meaning Criteria and the Logic of Fuzzy Concept: In Paul Peranteau, judith Levi and Gloria Phrase (Eds.), papers from The Eight Regional Meeting, Chicago Linguistics Society (CLS 8), pp 183-228.

Lakoff, R. (1973). Language and woman's place. Language in Society, 2, 45-79. 
Lakoff, R. (1975). Language and women's place. New York: Harper and Row.

Nemati, M.L., \& Bayer, J.M. (2007). Gender Differences in the Use of Linguistics Form: A Comparative Study of Persian and English. South Asian Language Review, 9 (2), 30-38.

Serholt, S. (2012). Hedges and Booster in Academic Writing: A Study of Gender Differences in Essays Written by Swedish Advanced Learners of English. Goteborgs Unversitat.

Swales, J. (1990). Genre Analysis. Cambridge: Cambridge University Press.

Varttala, T.A. (2001). Hedging in Scientifically Oriented Discourse: Exploring Variation According to Discipline and Intended Audience. Unpublished $\mathrm{PhD}$ dissertation. University of Tampereen Yliopisto, Finland.

Ygiz, O. \& Demir, C., (2014) Hedging Strategies in Academic Discourse: A Comparative Analysis of Turkish Ariters and Native Writers of English. Procedia: Science and Behavioral Science 260-268. 